\title{
Correction
}

Hautarzt 2018 $69: 92-94$

https://doi.org/10.1007/s00105-017-4102-5

Online publiziert: 13. Dezember 2017

(c) Springer Medizin Verlag $\mathrm{GmbH}$, ein Teil von Springer Nature 2017

CrossMark
T. Silber · K. Schweinzer · A. Strölin

Phlebologie, Universitäts-Hautklinik Tübingen, Tübingen, Deutschland

\section{Correction: Diagnostik und Therapie akuter venöser Erkrankungen}

\section{Correction:}

Hautarzt 2017

https://doi.org/10.1007/s00105-017-

4006-4

Sehr geehrte Leserin, sehr geehrter Leser,

in - Tab. 2 des oben genannten Beitrags ist leider ein Fehler unterlaufen. Die Erhaltungsdosierung von Rivaroxaban (Xarelto ${ }^{\circledR}$ ) ab Tag 22 beträgt $20 \mathrm{mg}$ 1-mal/Tag. Die korrigierte Tabelle finden Sie im Folgenden.

Wir bitten Sie, zukünftig die korrigierte Version der Tabelle zu berücksichtigen und den Fehler zu entschuldigen.

\section{Korrespondenzadresse}

\section{Dr. T. Silber}

Phlebologie, Universitäts-Hautklinik Tübingen

Liebermeisterstr. 25, 72076 Tübingen,

Deutschland

toni.silber@med.uni-tuebingen.de

\section{Literatur}

11. Fachgesellschaft: F, Gefäßmedizin DGfAGf. S2Leitlinie: Diagnostik und Therapie der Venenthrombose und der Lungenembolie. AWMFLeitlinien-Register-Nr.065/002 2015. 
Tab. 2 Initiale Antikoagulation und Erhaltungstherapie bei Venenthrombose/Lungenembolie (3 bis 6 Monate nach Ereignis) -Stand:September 2015 [1]

\begin{tabular}{|c|c|c|c|c|}
\hline Wirkstoff/-gruppe & Präparat & Initiale Dosierung & Erhaltungsdosierung & $\begin{array}{l}\text { Anwendung/ } \\
\text { Besonderheiten }\end{array}$ \\
\hline \multicolumn{5}{|c|}{ Niedermolekulare Heparine } \\
\hline Certoparin & $\begin{array}{l}\text { Mono-Embolex } \\
8000 \text { I.E. Therapie }\end{array}$ & $8000 \mathrm{IE}$ & $8000 \mathrm{IE}^{\mathrm{a}}$ & 2-mal s. c./Tag \\
\hline Dalteparin & Fragmin $^{\circledR}$ & $\begin{array}{l}100 \mathrm{IE} / \mathrm{kg} \mathrm{KG} \\
200 \mathrm{IE} / \mathrm{kg} \mathrm{KG}\end{array}$ & $\begin{array}{l}100 \mathrm{IE} / \mathrm{kg} \mathrm{KG}^{\mathrm{a}} \\
200 \mathrm{IE} / \mathrm{kg} \mathrm{KG}^{\mathrm{a}}\end{array}$ & $\begin{array}{l}\text { 2-mal s. c./Tag } \\
1 \text {-mal s. c./Tag }\end{array}$ \\
\hline Enoxaparin ${ }^{b}$ & Clexane ${ }^{\circledR}$ & $1,0 \mathrm{mg} / \mathrm{kg} \mathrm{KG}$ & $1,0 \mathrm{mg} / \mathrm{kg} \mathrm{KG}^{\mathrm{a}}$ & 2-mal s. c./Tag \\
\hline Nadroparin & $\begin{array}{l}\text { Fraxiparine }{ }^{\circledR} \\
\text { Fraxodi }^{\circledR}\end{array}$ & $\begin{array}{l}0,1 \mathrm{ml} / 10 \mathrm{~kg} \mathrm{KG} \\
0,1 \mathrm{ml} / 10 \mathrm{~kg} \mathrm{KG}\end{array}$ & $\begin{array}{l}0,1 \mathrm{ml} / 10 \mathrm{~kg} \mathrm{KG}^{\mathrm{a}} \\
0,1 \mathrm{ml} / 10 \mathrm{~kg} \mathrm{KG}^{\mathrm{a}}\end{array}$ & $\begin{array}{l}\text { 2-mal s. c./Tag } \\
\text { 1-mal s.c./Tag }\end{array}$ \\
\hline Reviparin & $\begin{array}{l}\text { Clivarin }^{\circledR} \\
\text { Clivarodi }^{\circledR}\end{array}$ & $\begin{array}{l}0,6 \mathrm{ml} \text { bei } K G 45-60 \mathrm{~kg} \\
0,6 \mathrm{ml} \text { bei } \mathrm{KG}>60 \mathrm{~kg}\end{array}$ & $\begin{array}{l}0,6 \mathrm{ml} \text { bei KG } 45-60 \mathrm{~kg}^{\mathrm{a}} \\
0,6 \mathrm{ml} \text { bei KG }>60 \mathrm{~kg}^{\mathrm{a}}\end{array}$ & $\begin{array}{l}\text { 2-mal s. c./Tag } \\
\text { 1-mal s. c./Tag }\end{array}$ \\
\hline Tinzaparin & Innohep ${ }^{\circledR}$ & $175 \mathrm{IE} / \mathrm{kg} \mathrm{KG}$ & $175 \mathrm{IE} / \mathrm{kg} \mathrm{KG}^{\mathrm{a}}$ & 1-mal s. c./Tag \\
\hline \multicolumn{5}{|l|}{ Pentasaccharid } \\
\hline Fondaparinux & Arixtra $^{\circledR}$ & $\begin{array}{l}7,5 \mathrm{mg} \\
5 \mathrm{mg} \text { bei } \mathrm{KG}<50 \mathrm{~kg} \\
10 \mathrm{mg} \text { bei } \mathrm{KG}>100 \mathrm{~kg}\end{array}$ & $\begin{array}{l}7,5 \mathrm{mg}^{\mathrm{a}} \\
5 \mathrm{mg} \text { bei } K G<50 \mathrm{~kg} \\
10 \mathrm{mg} \text { bei } \mathrm{KG}>100 \mathrm{~kg}\end{array}$ & 1-mal s. c./Tag \\
\hline \multicolumn{5}{|c|}{ Unfraktionierte Heparine } \\
\hline $\begin{array}{l}\text { Heparin-Kalzium } \\
\text { Heparin-Natrium }\end{array}$ & $\begin{array}{l}\text { Heparin-Calcium }{ }^{\circledR} \\
\text { Heparin-Natrium }^{\circledR}\end{array}$ & Bolus $5000 \mathrm{IE}$, dann $15-20 \mathrm{IE} / \mathrm{kg} \mathrm{KG/h}$ & $\begin{array}{l}\text { Ca. } 15-20 \mathrm{IE} / \mathrm{kg} \mathrm{KG} / \mathrm{h}^{\mathrm{a}} \\
\text { aPTT-gesteuert }\end{array}$ & $\begin{array}{l}\text { Bolus i. v., dann i. v.-Infusion } \\
\text { oder 2-mal s. c./Tag } \\
\text { aPTT-Kontrolle notwendig }\end{array}$ \\
\hline \multicolumn{5}{|c|}{ Direkte orale Antikoagulanzien } \\
\hline Dabigatrantexilat & Pradaxa ${ }^{\circledR}$ & NMH, UFH oder FDX mindestens 5 Tage & $150 \mathrm{mg}$ & 2-mal oral/Tag \\
\hline Rivaroxaban & Xarelto ${ }^{\circledR}$ & $15 \mathrm{mg}$ 2-mal/Tag oral 21 Tage & Ab 22. Tag $20 \mathrm{mg} 1-\mathrm{mal} / \mathrm{Tag}$ & - \\
\hline Apixaban & Eliquis ${ }^{\circledR}$ & 10 mg 2-mal/Tag oral 7 Tage & Ab 8. Tag 5 mg 2-mal/Tag & - \\
\hline Edoxaban & Lixiana $^{\circledR}$ & NMH, UFH oder FDX mindestens 5 Tage & $60 \mathrm{mg}$ & 1-mal oral/Tag \\
\hline \multicolumn{5}{|c|}{ Vitamin-K-Antagonisten $^{c}$} \\
\hline Phenprocoumon & $\begin{array}{l}\text { Marcumar }^{\circledR} \\
\text { Falithrom }^{\circledR}\end{array}$ & $\begin{array}{l}\text { NMH, UFH oder FDX parallel bis INR } \\
\geq 2,0 \text { an } 2 \text { aufeinanderfolgenden Tagen }\end{array}$ & $\begin{array}{l}\text { INR-gesteuert } \\
\text { Ca. } 1,5-4,5 \mathrm{mg}\end{array}$ & $\begin{array}{l}\text { 1-mal oral/Tag } \\
\text { INR-Zielbereich zwischen } 2 \\
\text { und } 3\end{array}$ \\
\hline Warfarin-Natrium & Coumadin $^{\circledR} 5 \mathrm{mg}$ & $\begin{array}{l}\text { NMH, UFH oder FDX parallel bis INR } \\
\geq 2,0 \text { an } 2 \text { aufeinanderfolgenden Tagen }\end{array}$ & $\begin{array}{l}\text { INR-gesteuert } \\
\text { Ca. 2,5-10 mg }\end{array}$ & $\begin{array}{l}\text { 1-mal oral/Tag } \\
\text { INR-Zielbereich zwischen } 2 \\
\text { und } 3\end{array}$ \\
\hline \multicolumn{5}{|c|}{$\begin{array}{l}\text { Vor Therapiebeginn sind die Fachinformationen der Hersteller zu beachten, insbesondere wegen der vorgegebenen Dosisreduktionen in Risikogruppen } \\
\text { aPTT aktivierte partielle Thromboplastinzeit, FDX Fondaparinux, IE internationale Einheiten, INR „International Normalized Ratio", i. v. intravenös, KG Kör- } \\
\text { pergewicht, NMH niedermolekulare Heparine, s. c. subkutan, UFH unfraktionierte Heparine } \\
\text { "Präparatespezifische Zulassungssituation beachten } \\
\text { 'Enoxaparin mit 1,5 mg/kg KG/Tag } \\
\left.\text { 'Acenocoumarol (Sintrom }{ }^{\circledR}\right) \text { zugelassen in Österreich/der Schweiz }\end{array}$} \\
\hline
\end{tabular}


Hier steht eine Anzeige.

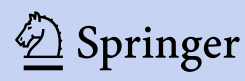

